\title{
Computational Modelling of Multi-field Ionic Continuum Systems
}

\author{
John Michopoulos \\ Special Projects Group, Code 6303, Naval Research Laboratory, U.S.A. \\ john.michopoulos@nrl.navy.mil
}

\begin{abstract}
The present paper presents a methodology for modelling the behavior of continua under multi-field influence, along with the architecture of its computational implementation. It is being applied for the special case of modelling multi-field ionic conductive material systems. Its computational implementation addresses generation and solution of both the constitutive and the field evolution equations. An application of this methodology for the case of electric multi-hygrothermoelasticity generates a model for materials participating in artificial muscle applications. The corresponding system of nonlinear governing partial differential equations describing the state evolution of large deflection plates made from such materials is derived as an example. Finally, initial numerical solution examples of these electro-hygro-thermally generalized Von-Karman equations are presented.
\end{abstract}

\section{Introduction}

The present paper reports on the developmental startup of a computational and algorithmic infrastructure that attempts not to ignore any coupled field and/or transport effects nor it makes any geometric simplifications while it captures the behavioral modelling of associated continuum systems.

The computational implementation and application examples on ionic artificial muscle materials are part of a validation effort at the sub-component level of a more general data-driven environment for multiphysics applications (DDEMA) that has been preliminarily described elsewhere [1/23].

Recent progress on processing and development of various multi-field activated materials such as electro-active polymers (EAP) and ionic polymer conductive composites (IPCC) for artificial muscle applications has underlined the general need for a rigorous, complete and preferably automated modelling of their behavior from a continuum coupled multi-field perspective.

There are various generalized approaches for deriving multi-field theories 4 5.67] in the 4D space-time domain. Furthermore, mass transport considerations were combined with continuum theories based on continuous thermodynamics 8,9. In the continuum mechanics context, the governing partial differential equations (PDEs) can be produced when all constitutive equations have been eliminated through term rewriting of the conservation laws. 
Arguably, the goal of developing a general methodology to utilize computational technologies for automating the process of generating and utilizing multifield theories for demanding applications, has not been reached. The plethora of analytic approaches as well as the pluralism, the quick evolution, and the heterogeneity of the available software and hardware infrastructure was a significant set of reasons that is contributing to this failure.

The novelty of this paper is that it attempts to address these difficulties by presenting both an analytical activity workflow along with an architecture of a computational infrastructure to derive and solve the equational system representing the behavior of multi-field ionic systems.

The paper presents the abstract algorithmic context of the general modelling process in Section 2, from the perspective of continuous multi-field constitutive theories (CFTs). Section 3 presents the general activity workflow of this process along with a computational architecture of a computational infrastructure that implements this workflow. Application of this methodology produced the generalized Von-Karman partial differential equations (PDEs) presented in Section 4 along with a solution example. Conclusions provide the closure of the paper.

\section{Multi-field Model Derivation}

Every deformable continuum under multi-field generalized loading including reactive substance and charge diffusion can be considered as a system that can be described in space and time by the evolution of its state variables. Some of these variables can be thought of as dependent or output parameters and some as independent or input parameters. A systemic abstraction of such a medium is presented in Fig. (1a) and its corresponding continuum one in Fig. (1b). The systemic abstraction differentiates between the bulk material state behavior and the structural state behavior, while the continuum mechanics traditional approach never makes this differentiation. Constitutive behavior refers to the bulk material state behavior (in the sense of the representative volume element behavior), while systemic behavior is the composition of both the bulk material state behavior with the structural material state behavior.

The bulk behavior of such a system is usually described as a set of relational restrictions $\tilde{F}$ among the state variables selected by the modeler as the ones pertinent to the situation at hand, given by

$$
\tilde{F}(\tilde{q}, \tilde{\xi}, \tilde{p})=0
$$

where $\tilde{p}, \tilde{\xi}$ and $\tilde{q}$ represent the input state variables, the internal state variables and the output state variables respectively. For those cases that these relations can be solved with respect to the output variables they are called constitutive relations of the form

$$
\tilde{q}=\tilde{C}(\tilde{\xi}, \tilde{p}) .
$$

Functionals $\tilde{C}$ in equation (2) represent an a-priori definable multi-functional mapping of the form $\mathrm{R}^{\operatorname{dim}(\tilde{p})} \times \mathrm{R}^{\operatorname{dim}(\tilde{\xi})} \stackrel{\tilde{C}}{\longrightarrow} \mathrm{R}^{\operatorname{dim}(\tilde{q})}$. In most path-history independent state spaces these functionals can be recovered by differentiation of 


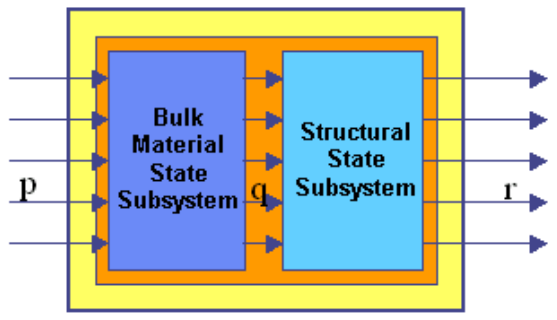

(a)

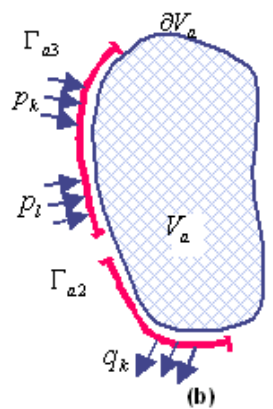

(b)

Fig. 1. Systemic (a) and continuum-based (b) descriptions of multi-field systems

an also a-priori definable potential function $\Xi(\tilde{\xi}, \tilde{p})$, with respect of the input (independent) state variables. This potential function has to be constructed as a function of the input and the internal state variables and should it be necessary any time derivatives of them. This can be expressed by,

$$
\tilde{q}=\nabla_{\tilde{p}} \Xi\left(\tilde{\xi}, \tilde{p}, \tilde{p}_{, t^{n}}, \tilde{\xi}_{, t^{n}}\right)
$$

This formalism imposes a conjugation between input and output state variables in a way that allows us to form "cause-effect" pairs $\left\{q_{i}, p_{i}\right\}$ that have the property that their product has unit dimensionality of energy density per unit of volume or mass. Various researchers have suggested various choices for the potential function required for the constitutive relations. The most practicable and well known among them are those of internal energy, enthalpy, Helmholtz free energy and Gibbs free energy.

In the context of continuous multiphysics, system behavior in terms of state evolution in most continuum systems, is expressed in terms of solutions of partial differential equations (PDEs) that enforce special topological form on the fields described by the spatial and time distributions of the state variables and further restrict the potential values these variables can take, i.e.

$$
\aleph_{i}\left(\nabla^{n} \tilde{q}, \tilde{q}_{, t^{m}}, \ldots\right)=0
$$

The traditional sources of such equations are the so-called "conservation" or "balance" laws of physics. These are the thermomechanical laws of conservation of mass, momentum, moment of momentum, energy, entropy flaw and the electrodynamic laws of conservation of electric displacement (Gauss-Faraday law), magnetic flux, electric charge, rotation of electric intensity (Faraday's law), magnetic intensity (Ampere's law). These are the axioms of continuous physics (ACP) that are not (formally) provable (in their most general form) but rather they are beliefs that we a priori accept to be true. Unfortunately they are not always enough for completely determining the state variable field evolution. For this reason the constitutive functionals are used to provide algebraic closure. Introducing the constitutive equations (2) or (3) into (4) and eliminating the 
independent variables or more generally half of the conjugate variables, yields a complete set of PDEs that constitute the so-called field equations of the system. They are of the form,

$$
\wp_{i}\left(\tilde{p}, \tilde{p}, t^{m}, \ldots\right)=0
$$

The ACP have been historically expressed in either their global (integral),

$$
\left(\int_{V} \phi \partial v\right)_{, t}=\int_{\partial V} \tau\{\phi\} \partial a+\int_{V} g\{\phi\} \partial v
$$

or the local (differential) form of a generalized divergence theorem [11] transformed via the Gauss-Ostrogratsky theorem,

$$
\phi_{, t}+\nabla \cdot(\boldsymbol{v} \phi)=\nabla \cdot \tau\{\phi\}+g\{\phi\},
$$

where $\phi$ is the field in a volume $V, \tau\{\phi\}$ is the influx of $\phi$ through the surface $\partial V$, and $g\{\phi\}$ is the amount of $\phi$ produced in the body (source term). Further simplifications on the form of the ACP can be obtained by applying some or all of the additional axiomatic and meta-axiomatic restrictions that are traditionally called the axioms of constitutive theory [7].

\section{Computational Workflow and Infrastructure}

The general process of simulating the behavior of continuum system usually involves the solution of the PDEs describing its space and time evolution via application of discrimination method over the domain of their applicability and the subsequent solution of a set of ordinary differential and eventually algebraic equations. However, as our ability to produce new material systems has outpaced our ability to model them, the need for automating the process of deriving these PDEs as well as the prerequisite sub-models and technologies has become apparent. In attempting to address this need we have developed a conceptual model of the workflow involved for developing a system of governing multi-field coupled PDEs. Figure 2 shows the activities and their interconnection along with the computational context they are embedded.

The essential features of this diagram capture the outline of the methodology described in the previous section. A crucial aim of the present effort is to extend the symbolic computing context as much to the left as possible and seamlessly integrate all contexts with each-other.

The current state of evolution of a continuously evolving computational infrastructure that implements the workflow of Fig. 2 is shown schematically in Fig. 3.

Lack of space does not allow detailed description of all modules and relation involved. However, the reader can easily follow the logic involved and recognize the programming languages involved. It is essential to underscore that $\mathrm{J} / \mathrm{Link}$ is the Mathematica 10 to Java and Java to Mathematica application programming interface. MathTensor [11] and Theorema [12] are packages developed to run under Mathematica, addressing the tensor manipulation and theorem proving needs of deriving the constitutive and field equations of the model at hand. 


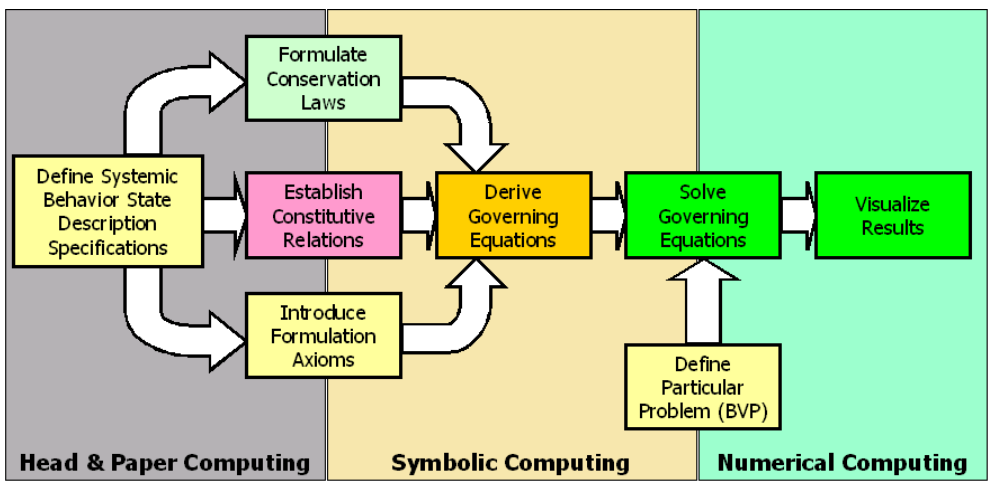

Fig. 2. Workflow diagram of activities and their interconnectivity along with their computational embedding for the process of developing multi-field models of continua

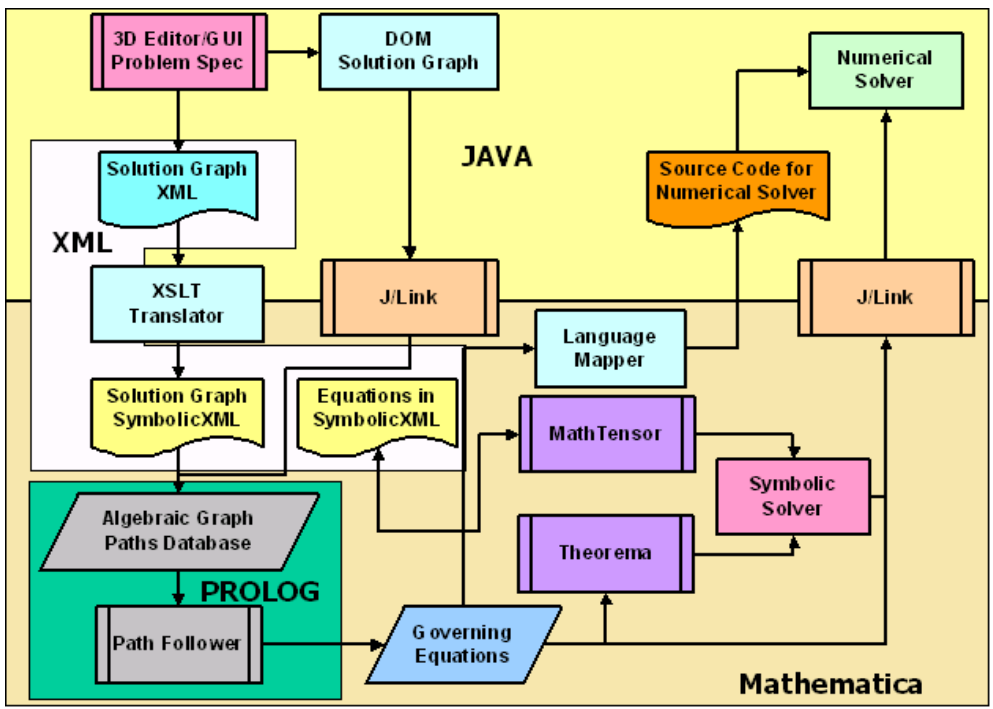

Fig. 3. Abstract architecture of computational infrastructure for implementing the workflow of Fig. 2

\section{Multi-component Hygro-Thermo-Electro-Elastic Plates}

Ionic material and structural systems that can be used as artificial muscles are falling within the category of multi-field systems. Limited electromechanical modelling of such systems has been already attempted for simple membrane and one dimensional systems and never considered the global continuous multi-field perspective of more than two simultaneously acting fields.

Application of the general process as described in the previous sections for the case of homogeneous mechanically isotropic system generated a set of nine 
field governing coupled PDEs and associated constitutive relations that have been originally derived elsewhere 1314 manually.

Introduction of Lagrange strains to account for large deformations along with insignificant ionic currents and chemical reactivity for multi-component diffusion, electric potential, temperature and mechanical deformation generalized loading yields the following set of modified Von-Karman nonlinear PDEs [16 17]:

$$
\begin{gathered}
\nabla^{2} \nabla^{2} w+(1+\nu) \sum_{k} \nabla^{2} X^{k}=\frac{h}{N}\left(\frac{q}{h}+F_{, 22} w_{, 11}-2 F_{, 12} w_{, 12}+F_{, 11} w_{, 22}\right), \\
\nabla^{2} \nabla^{2} F+E \sum_{k} \nabla^{2} X^{k}=E\left[\left(w_{, 12}\right)^{2}-w_{, 11} w_{, 22}\right], \\
\frac{1-\nu}{2 E}\left(\nabla^{2} \delta_{i j}-\partial_{i} \partial_{j}\right) F \delta_{i j}+\nabla^{2} X^{k}=0 .
\end{gathered}
$$

Here $w, F, X^{k}$ are the deflection, the Airy stress function and the additional generalized field state variables and $\delta_{i j}, \nu, E, h, N, q$ are Kronecker's delta, Poisson's ratio, the modulus of elasticity, the plate thickness, the flexural rigidity and the distributed load respectively.

Equation (10) provides the closure of the system of equations (8) and (9) governs the balance of the generalized additional scalar fields $X^{k}[1314$ (and appears in its expected divergent theorem form).

Since there is no known closed form solution of the generalized Von-Karman equations an approximate solution based on Finite Element Analysis (FEA) can be utilized. The general purpose code flexPDE 15 was used for this task.

Although we solved various cases of boundary conditions due to the space limitations here, we will only present the case of a simply supported plate along all the edges with no lateral mechanical load. The value of $X^{k}=$ Temp at the boundary varies sinusoidally in time.

Figure 4 shows the distribution of deflection over the entire domain for increment 36 (4a) near the beginning of the cycle, and increment 121 (4b) on the end of the complete cycle. Clearly these two distributions verify the reversible actions of the applied field since the plate evolves from an all-negative to an all-positive deflection field.

Much is already known about how deflection and Airy function relate to each other from the traditional large deflection Von-Karman plate analysis [16 17]. All other fields and fluxes can be trivially computed by exploitation of the constitutive and flux definition relations given elsewhere [13:14] and are not provided here due to lack of space. The intension of this section is not to detail the derivation and solution methodologies for the corresponding PDEs but rather to provide evidence that is possible.

\section{Conclusions}

In this effort, we have described an abstract framework for multi-field modelling of material systems with emphasis to ionic materials used for artificial muscle 

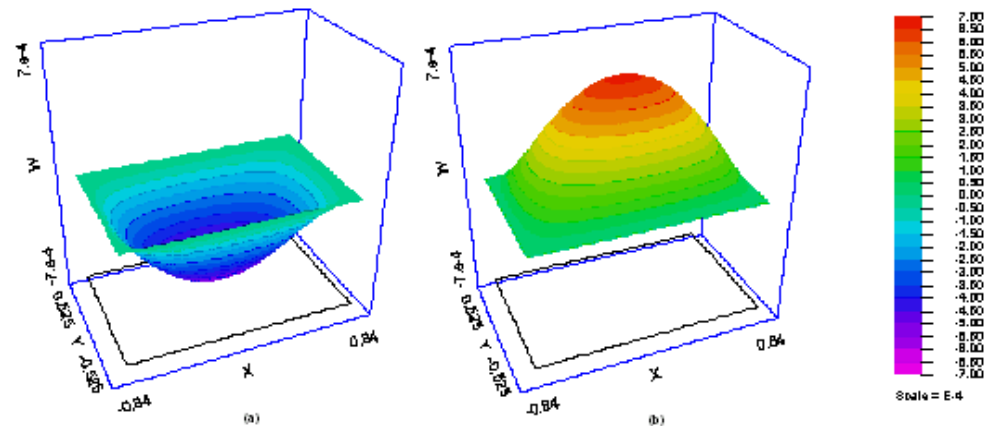

Fig. 4. Two instances of the deflection solution of a non-linear generalized Von-Karman system of PDEs governing a rectangular plate made from an ionic material

applications. Furthermore an abstract workflow of activities has been created to employ this framework. The current status of a computational infrastructure that attempts to instantiate this workflow is also briefly described. Application of this methodology and workflow has been utilized for deriving the generalized VonKarman equations for multi-field activation of large deflection plates. Finally an example of numerically solving the derived system is presented.

The approach followed still leaves open the issue of determination of the required constants participating in this formulation. Subsequent work in addition to evolving the computational infrastructure, will focus on this task.

Acknowledgement. The author acknowledges the support by the National Science Foundation under grant ITR-0205663 and professor M. Shahinpoor from the University of New Mexico, for his donation of ionic polymer composite material samples.

\section{References}

1. Michopoulos, J., Tsompanopoulou, P., Houstis, E., Rice, J., Farhat, C., Lesoinne, M., Lechenault, F., DDEMA: A Data Driven Environment for Multiphysics Applications,in: Proceedings of International Conference of Computational Science ICCS'03, Sloot, P.M.A., et al. (Eds.) Melbourne Australia, June 2-4, LNCS 2660, Part IV, Springer-Verlag, Haidelberg, (2003) 309-318.

2. Michopoulos, J., Tsompanopoulou, P., Houstis, E., Rice, J., Farhat, C., Lesoinne, M., Lechenault, F., Design Architecture of a Data Driven Environment for Multiphysics Applications, in: Proceedings of DETC'03, ASME DETC2003/CIE Chicago IL, Sept. 2-6 2003, Paper No DETC2003/CIE-48268, (2003).

3. Michopoulos, J., Tsompanopoulou, P., Houstis, E., Farhat, C., Lesoinne, M., Rice, J., Joshi, A., On a Data Driven Environment for Multiphysics Applications, Future Generation Computer Systems, in-print (2004).

4. Truesdell, C., Toupin, R., 1960, "The Classical Field Theories", in Handbuch der Physik ( Herausgegeben con S. Flugge) III/1, Springer-Verlag, Berlin. 
5. Truesdell, C., Noll, W., 1965, "The Non-Linear Field Theories of Mechanics", Handbuch der Physik ( Herausgegeben con S. Flugge) Bd. III/3, Springer-Verlag, Berlin.

6. Green, A. E., Naghdi, P. M., 1995, "A unified procedure for construction of theories of deformable media. I. Classical continuum physics", Proc. Roy. Soc. London Ser. A 448 /1934, pp. 335-356.

7. Eringen, A.C., Maugin., G.A., 1990, "Electrodynamics of Continua I. Foundations and Solid Media." Springer-Verlag, New York.

8. Sih, G.C., Michopoulos, J.G., Chou S.C., 1986, "Hygrothermoelasticity", Martinus Nijhoff Publishers (now Kluwer Academic).

9. Michopoulos, J.G., Sih, G.C., 1984, "Coupled Theory of Temperature Moisture Deformation and Electromagnetic Fields", Institute of Fracture and Solid Mechanics report IFSM-84-123, Lehigh University.

10. Wolfram, S.,1999, "The Mathematica Book", 4th Edition, Wolfram Media, Cahmpaign and Cambridge University Press, Cambridge.

11. Parker, L., Christensen, S. M., 1994, "MathTensor: A System for Doing Tensor Analysis by Computer", Addison-Wesley, Reading.

12. Buchberger B. 1998, "Theorema: Computer-Supported Mathematical Proving", in proc. of Annual Meeting of the Mathematics Department of the University of Wales, Gregynod.

13. Michopoulos, J.G., Shahinpoor, M., 2002, "Towards a Multiphysics Formulation of Electroactive Large Deflection Plates Made from Ionic Polymeric Artificial Muscles", Proceedings of First World Congress on Biomimetics and Artificial Muscles, 9-11 December 2002, Albuquerque NM

14. Michopoulos, J., 2003, "Numerical Solution of the Multiphysics Generalized Von-Karman Equations for Large Deflection Ionic Polymer Plates", in: Proc. of DETC'03, ASME DETC2003/CIE Chicago IL, Sept. 2-6 2003, Paper No DETC2003/CIE-48207.

15. PDE Solutions Inc., "FlexPDE 3 Reference manual", PDE Solutions Inc., (2001).

16. vonKarman, T., 1910, "Festifkeitsprobleme im Maschinebau", Encyk. der Math. Wiss., pp. 349.

17. Levy, S., 1942, "Bending of Rectangular Plates with Large Deflections", NACA Technical Report No. 737. 